\title{
Closely Related Poleroviruses Depend on Distinct Translation Initiation Factors to Infect Arabidopsis thaliana
}

\author{
C. Reinbold, ${ }^{1,2}$ S. Lacombe, ${ }^{3}$ V. Ziegler-Graff, ${ }^{4}$ D. Scheidecker, ${ }^{4}$ L. Wiss,,${ }^{1,2}$ M. Beuve,,${ }^{1,2}$ C. Caranta, ${ }^{3}$ and \\ V. Brault ${ }^{1,2}$ \\ ${ }^{1}$ INRA, UMR 1131 SVQV, 28 rue de Herrlisheim, F-68021 Colmar, France; ${ }^{2}$ Université de Strasbourg, 4 rue Blaise Pascal, \\ F-67081 Strasbourg, France; ${ }^{3}$ INRA, UR1052 GAFL, F-84143 Montfavet, France; ${ }^{4}$ CNRS, IBMP, UPR 2357, 12 rue du Général \\ Zimmer, F-67084 Strasbourg, France
}

Submitted 12 July 2012. Accepted 17 September 2012.

In addition to being essential for translation of eukaryotic mRNA, translation initiation factors are also key components of plant-virus interactions. In order to address the involvement of these factors in the infectious cycle of poleroviruses (aphid-transmitted, phloem-limited viruses), the accumulation of three poleroviruses was followed in Arabidopsis thaliana mutant lines impaired in the synthesis of translation initiation factors in the eIF4E and eIF4G families. We found that efficient accumulation of Turnip yellows virus (TuYV) in $A$. thaliana relies on the presence of eIF (iso)4G1, whereas Beet mild yellowing virus (BMYV) and Beet western yellows virus-USA (BWYV-USA) rely, instead, on eIF4E1. A role for these factors in the infectious processes of TuYV and BMYV was confirmed by direct interaction in yeast between these specific factors and the $5^{\prime}$ viral genomelinked protein of the related virus. Although the underlying molecular mechanism is still unknown, this study reveals a totally unforeseen situation in which closely related viruses belonging to the same genus use different translation initiation factors for efficient infection of $\boldsymbol{A}$. thaliana.

Turnip yellows virus (TuYV, formerly known as Beet western yellows virus-FL1), Beet mild yellowing virus (BMYV), and Beet western yellows virus-USA (BWYV-USA) belong to the Polerovirus genus in the Luteoviridae family. Poleroviruses are single-stranded RNA viruses encapsidated in icosahedral particles. In contrast to cellular mRNAs that possess a $\mathrm{m}^{7} \mathrm{G}$ cap structure at their $5^{\prime}$ end and a poly-A tail at their $3^{\prime}$ end, poleroviral RNAs have a viral genome-linked protein (VPg) covalently attached to the $5^{\prime}$ terminus and they lack a poly-A (Mayo and Ziegler-Graff 1996). Because poleroviruses exhibit a vascular tissue tropism, virus replication and movement, therefore, are limited to companion cells, phloem parenchyma cells, and sieve tubes (Mutterer et al. 1999).

Current address of S. Lacombe: IRD, UMR RPB (IRD-CIRAD-UM2), 911 Avenue Agropolis, F-34394 Montpellier, France.

Corresponding author: V. Brault; E-mail: veronique.brault@ colmar.inra.fr

* The $\boldsymbol{e}$-Xtra logo stands for "electronic extra" and indicates three supplementary figures and three supplementary tables are published online.

This article is in the public domain and not copyrightable. It may be freely reprinted with customary crediting of the source. The American Phytopathological Society, 2013.
In recent years, numerous studies on plant-virus interactions have identified eIF4E and eIF4G proteins from the translation initiation complex as key components required for plant susceptibility to a wide range of RNA viruses (Le Gall et al. 2011; Robaglia and Caranta 2006). During mRNA translation, eIF4E provides the cap-binding function and is associated with the scaffolding protein eIF4G to form the eIF4F complex (Goodfellow and Roberts 2008; Marcotrigiano et al. 1999). Plants possess a second eIF4F complex (referred to as eIF(iso)4F) which results from the association between eIF(iso) $4 \mathrm{E}$ and eIF(iso)4G isoforms (Gallie and Browning 2001). Plant genes encoding proteins of the eIF4F complexes belong to small multigenic families. In Arabidopsis thaliana, three genes code for proteins of the eIF4E family (eIF4E1, $e I F 4 E 2$, and $e I F 4 E 3$ ), one gene codes for $e I F(i s o) 4 E$, and one for a noncanonical eIF4E-like protein, known as a novel capbinding protein (nCBP). Only one gene codes for eIF4G and two for eIF(iso)4G subfamily (eIF(iso)4G1 and eIF(iso)4G2) (Robaglia and Caranta 2006).

A distinctive feature of eIF4E and eIF4G, in comparison with other host factors required for plant-virus interactions, is that they show natural polymorphism associated with recessive resistance in many crops. The eIF4E factor has been implicated in natural resistance to several potyviruses in diverse plant species (Le Gall et al. 2011) but also in barley resistance to a bymovirus (Kanyuka et al. 2005; Stein et al. 2005) and in melon resistance to a carmovirus (Nieto et al. 2006). In addition to eIF4E-mediated resistance, eIF(iso)4G1 and eIF4G were demonstrated to be involved in rice resistance to Rice yellow mottle virus (RYMV, genus Sobemovirus) and Rice tungro spherical virus (genus Waikavirus), respectively (Albar et al. 2006; Hebrard et al. 2010; Lee et al. 2010). Knock-out $A$. thaliana mutants for eIF4G or eIF(iso) $4 \mathrm{G}$ were also unable to support potyvirus infection, indicating that the whole eIF4F complex may be necessary for potyvirus infection (Gallois et al. 2010; Nicaise et al. 2007).

From the virus side, the VPg has been identified as the major determinant required to overcome virus resistance controlled by eIF4E or eIF4G factors (Hebrard et al. 2008; Le Gall et al. 2011; Truniger and Aranda 2009). In a few cases, however, other viral components function as the avirulence factor toward eIF4E: the C-terminal part of the cylindrical inclusion protein of Lettuce mosaic virus (LMV) (Abdul-Razzak et al. 2009), the $3^{\prime}$ untranslated region of Melon necrotic spot virus genome (Diaz et al. 2004), and the P3 protein of Pea seed-borne mosaic virus (Hjulsager et al. 2006). 
The molecular mechanisms underlying virus plant susceptibility mediated by eIF4E or eIF4G factors involve, for potyviruses, a physical interaction between eIF4E and the VPg (Beauchemin et al. 2007; Khan et al. 2006; Miyoshi et al. 2006; Wittmann et al. 1997) and, for RYMV, an interaction between eIF(iso)4G1 and the VPg (Hebrard et al. 2010).

In the Luteoviridae family, limited information is available on the requirement for translation initiation factors in the virus replication cycle. None of the eIF4F components has been identified as cellular factors influencing plant susceptibility to these viruses. The only implication of translation initiation factors was reported for Barley yellow dwarf virus (BYDV), a member of the Luteovirus genus, which, in contrast to members of the Polerovirus genus, does not possess a VPg. Treder and associates (2008) provided evidence that components of the eIF4F and eIF(iso)4F complexes bind to a $3^{\prime}$ cap-independent translation element, via a direct interaction with eIF4G. The eIF4F and eIF(iso) $4 \mathrm{~F}$ complexes are then delivered to the $5^{\prime}$ end of the genome by long-distance base pairing, to facilitate translation. eIF4E increases translation efficiency by interacting with eIF4G but is dispensable for this process, whereas eIF4G is required to initiate translation of uncapped RNA (Treder et al. 2008).

Altogether, these results underline the conserved role of translation initiation factors $4 \mathrm{E}$ or $4 \mathrm{G}$ in plant-virus interactions. However, many important questions remain to be investigated. In particular, can the requirement of these translation initiation factors be generalized to all VPg-linked RNA viruses? To determine whether poleroviruses, whose RNA genome is linked to a VPg at the $5^{\prime}$ end, also require translation initiation factors eIF4E or eIF4G for efficient plant infection, several $A$. thaliana mutant lines affected in the synthesis of the different components of the eIF4F complex were challenged with TuYV or BMYV, two closely related poleroviruses. Susceptibility analyses of the mutants showed that eIF(iso)4G1 is required for TuYV infection whereas a predominant role for eIF4E1 was observed for BMYV. Another polerovirus, closely related to BMYV, BWYV-USA, also requires eIF4E1 for its infectious cycle. Moreover, a direct interaction between the VPg of TuYV or BMYV and the translation initiation factor involved in their cycle was observed using the yeast two-hybrid assay. These results not only demonstrate the involvement of translation initiation factors in the infection cycle of poleroviruses but also provide the first demonstration that two closely related viruses from the same genus may need translation initiation factors from different families for their infection cycle.

\section{RESULTS}

\section{eIF(iso)4G1 and, to a lesser extent, eIF(iso)4G2 are required for TuYV infection in A. thaliana.}

Ten A. thaliana mutant lines disrupted in each of the different genes coding for eIF4E and eIF4G factors were challenged with TuYV. The mutant lines were T-DNA single-insertion lines inhibited in the expression of translation-initiation factors eIF4E1, eIF4E2, eIF4E3, eIF(iso)4E, eIF4G, eIF(iso)4G1, eIF(iso)4G2, and nCBP. In addition to these T-DNA mutants, an A. thaliana line bearing a point mutation in the eIF4E1 coding sequence resulting in a C-terminal truncation of the eIF4E1 protein (referred as to cum1.1) (Yoshii et al. 2004), and a line in which both isoforms of $4 \mathrm{G}$ were simultaneously knocked out (referred as to At-eIF(iso)4G1G2), were included in the experiments. Except for this double mutant that exhibited a severe growth defect (Lellis et al. 2010) when compared with wild-type Col0 plants, all the other lines grew similarly to the wild type Col0. Several of these A. thaliana mutants have already been assayed for their ability to sustain accumulation of different viruses (Le Gall et al. 2011).
When the different $A$. thaliana mutant lines were challenged with TuYV, a significant reduction in virus accumulation in plants affected in the expression of eIF(iso)4G1 was observed (Fig. 1A; Supplementary Table S1). In four independent experiments, virus accumulation assessed by double-antibody sandwich enzyme-linked immunosorbent assay (DAS-ELISA) at 21 days postinoculation in the At-eIF(iso)4G1 mutant was statistically lower when compared with virus accumulation in wildtype Col0 plants (Supplementary Fig. S1A). The reduced accumulation of TuYV was even more drastic in plants where both eIF(iso)4G isoforms were affected. No difference in virus accumulation was recorded in At-eIF(iso) $4 G 2$ or in At-eIF4G mutants (Fig. 1A). In all experiments, TuYV accumulation was detected in At-eIF4E1, cum1.1, At-eIF4E2, At-eIF4E3, At$e I F($ iso $) 4 E$, and $A t-n C B P$ at levels similar to those observed in Colo.

Because very low TuYV virus titers were detected in the AteIF(iso) $4 G 1 G 2$ double mutant, presence of the TuYV genome was additionally verified by quantitative reverse-transcriptase polymerase chain reaction (qRT-PCR). Levels of TuYV accumulation in A. thaliana lines affected in the expression of the $4 \mathrm{G}$ factors was quantified from RNA extracted from upper leaves of TuYV-inoculated At-eIF4G, At-eIF(iso)4Gl, AteIF(iso)4G2, and At-eIF(iso)4G1G2 and compared with Col0. The cycle threshold $(\mathrm{Ct})$ values from the qRT-PCR experiment were compared with values obtained from the known copy number of viral RNA. Whereas a similar number of TuYV genomes was observed in the At-eIF4G and At-eIF(iso)4G2 mutants (1.91E+06 and 2.03E+06 genomes per nanogram of RNA, respectively) when compared with Col0 (2.41E+06 genomes per nanograms of RNA), TuYV accumulated five times less in the At-eIF(iso)4G1 mutant (4.41E+05 genomes per nanograms of RNA) (Fig. 2). Reduction of virus titer was even more severe in the double At-eIF(iso)4G1G2 mutant, where TuYV accumulated 13 times less (1.77E+05 genomes per nanograms of RNA) than in wild-type Col0 (Fig. 2).

Altogether, the results obtained from TuYV accumulation in the different $A$. thaliana mutants strongly support the involvement of At-eIF(iso) $4 G 1$ in the virus infectious cycle. The fact that the double mutant accumulates less TuYV than the At-eIF (iso) $4 G 1$ mutant, although susceptibility of the At-eIF(iso)4G2 mutant was not reduced, suggest that this isoform is only used by TuYV in the absence of eIF(iso) $4 \mathrm{G} 1$, because it is less efficient.

\section{BMYV infection depends on eIF4E1.}

In order to investigate the behavior of a closely related polerovirus, similar infectivity assays were conducted with BMYV. Whereas TuYV and BMYV display moderate amino acid sequence homology for the proteins encoded by the first two open reading frames located at the $5^{\prime}$ end of the genome ( 24 and $34 \%$ for P0 and P1, respectively), the other viral proteins exhibit a high sequence homology between the two viruses (63 to $91 \%$ for P2 to P5) (Guilley et al. 1995). First of all, when compared with TuYV accumulation in the wild-type Col0, we observed that BMYV titer in these plants was reduced (Fig. 1B). Comparison of the dilution curves of both viruses indicates that the lower optical density values in BMYV-Col0 infected plants do not reflect reduced BMYV accumulation relative to TuYV but, rather, a weaker recognition by the antibodies used in the DAS-ELISA assay (Supplementary Fig. S2).

BMYV-susceptibility analyses of the A. thaliana mutants showed a reduced virus titer in the two lines affected in the synthesis of eIF4E1 (At-eIF4E1 and cum1.1) (Fig. 1B; Supplementary Table S2). In contrast, the level of virus accumulation in At-eIF4E2, At-eIF4E3, and At-nCBP mutants was similar to that in the wild-type Col0 (Fig. 1B) and was significantly 
higher in plants altered in the expression of the eIF(iso)4E factor (Fig. 1B). Although we observed a tendency toward reduced BMYV accumulation in the three At-eIF4G, At-eIF(iso)4G1, and At-eIF(iso)4G2 mutants when compared with Col0 (Fig. 1B), this reduction was not significant. However, BMYV accumulated at a significantly reduced level in the double mutant At-eIF(iso)4G1G2 (Fig. 1B). Although TuYV was barely detectable in this mutant, BMYV accumulation was low but substantially above the threshold level and, therefore, was not considered to require qRT-PCR for further detection.

Among poleroviruses, BMYV displays a host range similar to that of BWYV-USA (Beuve et al. 2008). Therefore, we analyzed the behavior of BWYV-USA in the mutant lines. Remarkably, the infection pattern observed for BWYV-USA-inoculated lines was highly similar to that of BMYV. Virus accumulation was significantly reduced in cuml.1 and At-eIF(iso)4G1G2 and no major difference in virus titer was observed in the other lines compared with Col0, except for At-eIF(iso)4E, which sustained a higher virus level when compared with Col0 (Fig. 1C; Supplementary Table S3). The apparent higher BMYV accumulation in At-eIF4E3 was not significantly different from Col0.
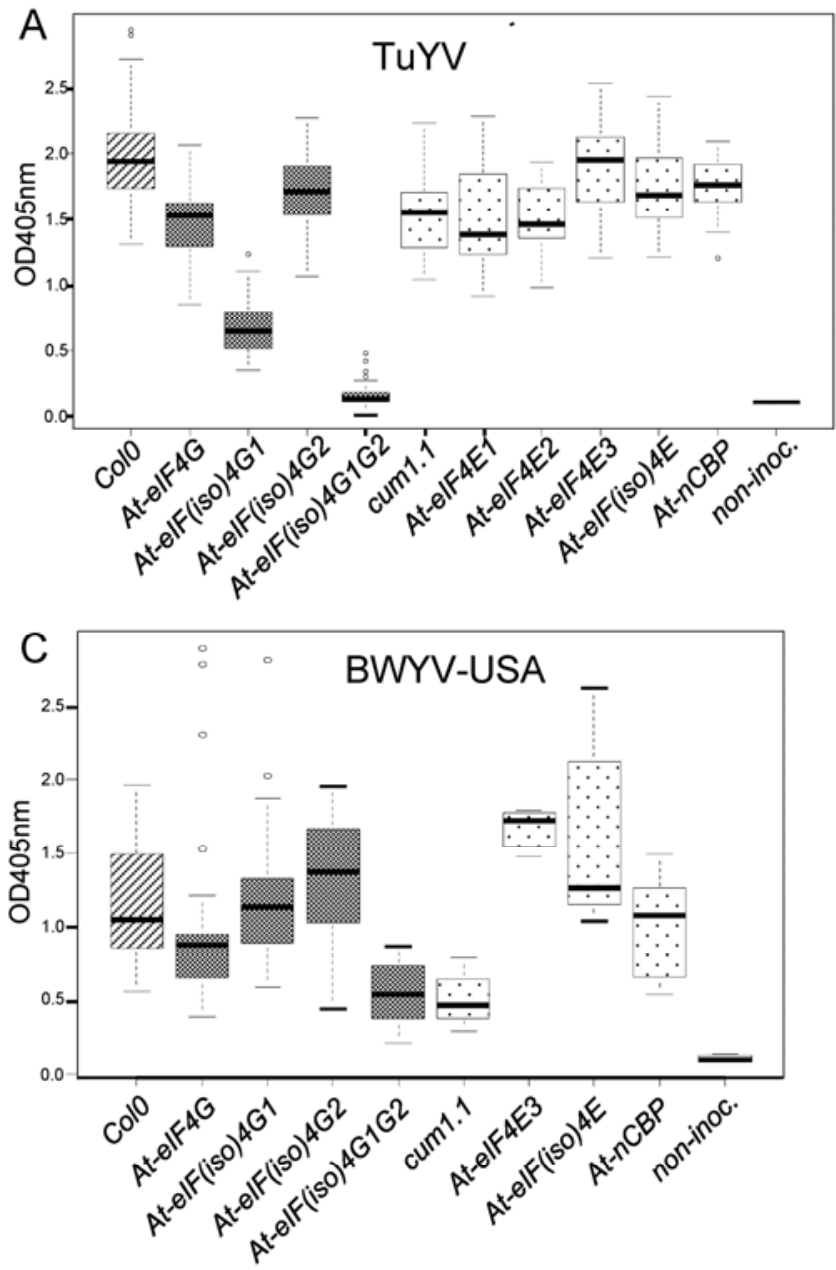

In contrast to TuYV, accumulation of BMYV and BWYVUSA in the different $A$. thaliana mutant lines highlighted a major involvement of eIF4E1 in both virus infections. In addition, accumulation of both viruses was severely reduced in the double At-eIF(iso)4G1G2 mutant while such a strong effect was not observed in the single mutants. This suggests that these factors act redundantly in BMYV and BWYV-USA infection cycles.

\section{TuYV-VPg and BMYV-VPg differ by their interaction patterns with eIF4E and eIF4G factors.}

To determine whether eIF4E or eIF4G factors interact physically with the VPg of poleroviruses, and if a correlation with successful viral infection can be drawn, the yeast two-hybrid system was used to monitor protein-protein interaction patterns between the different eIF4E and eIF4G proteins and TuYV- and BMYV-VPg. TuYV- and BMYV-VPg coding sequences have not been positioned with confidence on the viral genome of both viruses. However, based on sequence homology with the Potato leafroll polerovirus (PLRV), whose N terminus of the VPg has been identified by microsequencing (van

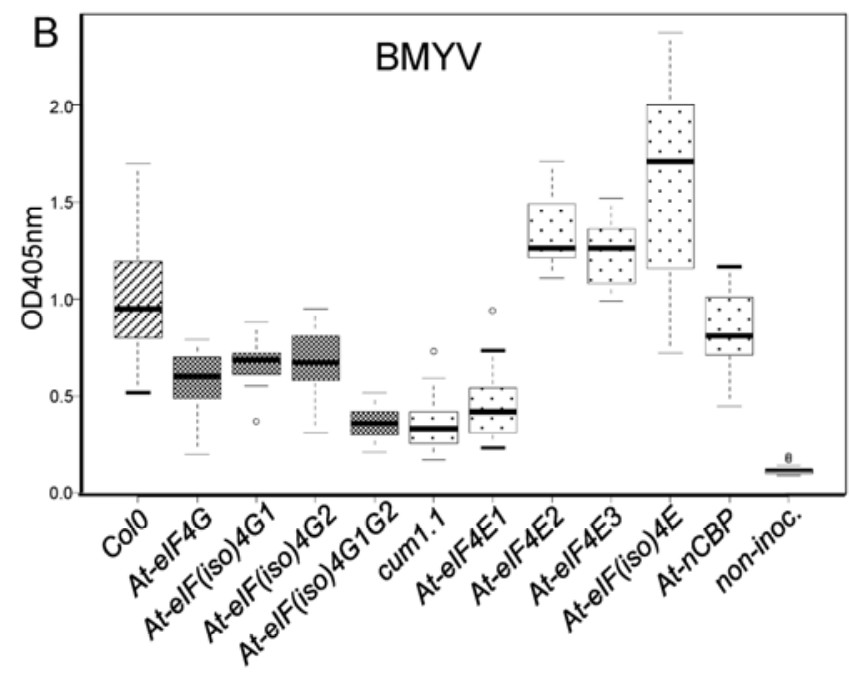

Fig. 1. Turnip yellows virus (TuYV), Beet mild yellowing virus (BMYV), and Beet western yellows virus-USA (BWYV-USA) accumulation in Arabidopsis thaliana mutants affected in the synthesis of translation initiation factors. Box and whisker representation of the data collected after plant inoculation with $\mathbf{A}$, TuYV; B, BMYV; or C, BWYV-USA. A. thaliana mutant lines-namely, At-eIF4G, At-eIF(iso)4G1, At-eIF(iso)4G2, At-eIF(iso)4G1G2, At-eIF4E1, cum1.1, At-eIF4E2, At-eIF4E3, At-eIF(iso)4E, and At-novel cap-binding protein ( $n C B P$ )-were inoculated with TuYV or BMYV using viruliferous aphids in 14 independent experiments (eight for TuYV and six for BMYV). Except for At-eIFE1 and At-eIF4E2, the same A. thaliana lines were aphid inoculated with BWYV-USA in five independent experiments. Col0 was used as a reference line. Virus accumulation in noninoculated leaves was measured by enzymelinked immunosorbent assay 3 weeks postinoculation (optical density [OD] at $405 \mathrm{~nm}$ ). End of the box represents the upper and lower quartiles. The median is marked by a horizontal line inside the box. Whiskers are the two lines outside the box that extend to the highest and lowest observations; $\circ=$ outlier and non-inoc. $=$ noninoculated plants from the different lines. The graph was constructed using $\mathrm{R}$ commander. 
der Wilk et al. 1997), the VPg-encoding sequences of TuYV and BMYV were located in the central domain of the P1 sequence downstream of the putative serine-like proteinase domain (Fig. 3A). Assuming that maturation of the VPg occurs through an internal proteolytic cleavage of $\mathrm{P} 1$, the $5^{\prime}$ terminal nucleotide of the TuYV- and BMYV-VPg coding regions is predicted to be at position 1,371 and 1,372 of their respective genomes. The $3^{\prime}$ end of the VPg-encoding sequence was assigned to nucleotide 1,544 for TuYV, and 1,542 for BMYV, assuming that both TuYV- and BMYV-VPg have a size similar to that predicted for PLRV which was estimated to be approximately 7 kDa (Fig. 3A) (Mayo et al. 1982). The C-terminal domain of the PLRV-P1 protein was detected in infected plants (Prüfer et al. 1999), suggesting that this cleavage product could also have a biological function in the polerovirus cycle. Therefore, constructs covering the $\mathrm{C}$-terminal domain of TuYV- or BMYV-P1 (P1- $\left.\mathrm{C}_{\text {term }}\right)$ and encompassing the putative VPg sequences were included in the analysis (Fig. 3A).

The ability of TuYV-VPg and TuYV-P1-C $\mathrm{C}_{\text {term }}$ to bind 4E factors was assessed by yeast two-hybrid assay, as described by Charron and associates (2008). eIF4E1-, eIF4E2-, eIF4E3-, eIF(iso)4E-, and nCBP-encoding sequences were cloned inframe with the GAL4 transcription activation domain (AD) into yeast pGADT7 vector, whereas the predicted TuYV-VPg and TuYV-P1- $\mathrm{C}_{\text {term }}$ encoding sequences were fused to the GAL4 DNA binding domain (BD) into the yeast pGBKT7 vector. After transformation and mating, yeast double transformants were plated onto medium lacking either histidine (-HWL) or histidine and adenine (-AHWL), allowing selection of yeast cells where interactions between the fusion proteins occurred.

Expression of TuYV-VPg from the pGBKT7 vector in cells cotransformed with the pGADT7 empty vector resulted in autonomous activation of the HIS3 reporter gene and allowed yeast growth on the selective medium (-HWL). However, autoactivation of the second reporter gene, $A D E 2$, by the TuYVVPg construct was not observed (i.e., absence of yeast growth on the medium [-AHWL]), allowing interaction tests with the $4 \mathrm{E}$ factors. Co-transformation of yeast cells with TuYV-VPg and the $4 \mathrm{E}$ factors did not result in any yeast development on

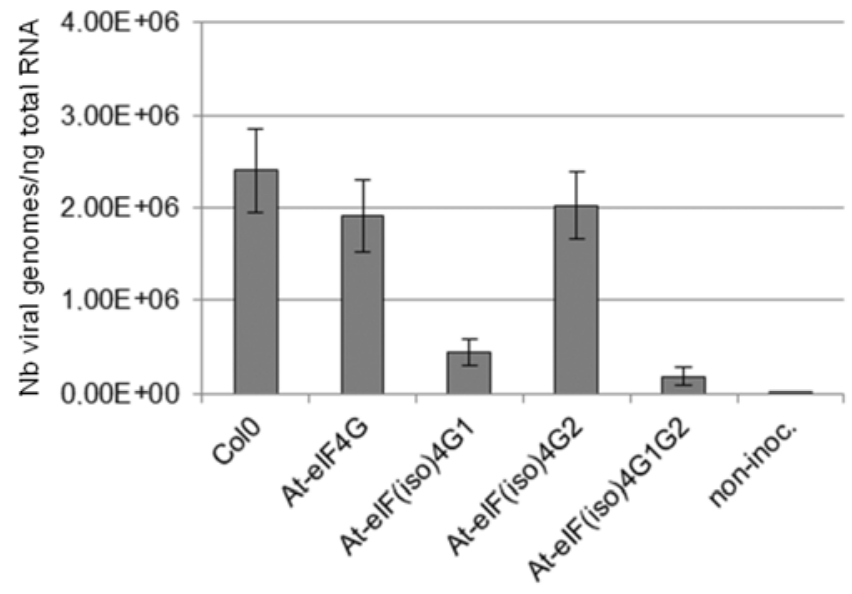

Fig. 2. Accumulation of Turnip yellows virus (TuYV) genomes in Arabidopsis thaliana mutants knocked out for eIF4G isoforms measured by quantitative reverse-transcription polymerase chain reaction. Number of virus genomes in the wild-type Col0 plants is compared with four A. thaliana mutants impaired in the expression of the translation initiation factors eIF4G, eIF(iso)4G1, eIF(iso)4G2, and both eIF(iso)4G1 and eIF(iso)4G2 (At-eIF(iso)4G1G2). Average number of viral genome copies and standard deviation were obtained from $6 \mathrm{Col0,} 6 \mathrm{At}$-eIF4G, 6 At-eIF(iso)4G1, $6 \mathrm{At}$ eIF(iso)4G2, and 12 At-eIF(iso)4G1G2 plants. non-inoc. = noninoculated plants from the different lines. the selective medium (-AHWL), suggesting the absence of a strong interaction (if any) between $4 \mathrm{E}$ factors and the TuYVVPg (Fig. 3B). Similarly, when TuYV-P1-C term was coexpressed with eIF4E factors, no interaction was observed (Fig. 3B).

To evaluate interactions of TuYV-VPg and TuYV-P1- $\mathrm{C}_{\text {term }}$ with the $4 \mathrm{G}$ factors, the viral coding sequences were cloned into pGADT7 to avoid autonomous transcription activity of the TuYV-VPg fused to the GAL4-BD. The 4G factor sequences were cloned into pGBKT7 vector and none of them, when expressed with the empty pGBKT7 vector, induced autonomous activation of both reporter genes (Fig. 3B). Co-expression in yeast cells of eIF(iso)4G1 with TuYV-VPg or TuYV-P1-C term allowed the cells to develop on the selective medium (-HWL), indicating an interaction between the partners (Fig. 3B). Moreover, yeast cells coexpressing TuYV-P1- $\mathrm{C}_{\text {term }}$ and eIF(iso)4G1 were able to grow on the more stringent medium (-AHWL), supporting a strong interaction between the two proteins. No interaction could be detected with the two other eIF4G proteins.

BMYV-VPg and BMYV-P1-C term protein interactions with $4 \mathrm{E}$ or $4 \mathrm{G}$ factors were tested following a procedure similar to the one described for TuYV. BMYV-VPg and BMYV-P1- $\mathrm{C}_{\text {term }}$ predictive sequences were cloned in fusion with GAL4-AD in pGADT7, while the 4E factors were expressed as GAL4-BD fusion proteins from pGBKT7. Autonomous activation by the $4 \mathrm{E}$ factors of the HIS3 reporter gene but not of the $A D E 2$ was observed (Fig. 3C). However, coexpression of the eIF4E1, eIF4E2, and eIF4E3 factors together with BMYV-VPg or BMYV-P1- $\mathrm{C}_{\text {term }}$ led to yeast growth on the stringent (-AHWL) medium, supporting interactions between both viral proteins and the three 4E factors (Fig. 3C).

To further address interactions of the $4 \mathrm{G}$ factors with BMYV-derived proteins, BMYV-VPg or BMYV-P1- $\mathrm{C}_{\text {term }}$ were expressed from the pGBKT7 vector, whereas $4 \mathrm{G}$ factors were produced from the pGADT7 vector. Expression of BMYV-VPg from pGBKT7 induced autoactivation of the HIS3 reporter gene and not of the $A D E 2$ gene. However, a faint yeast growth development was observed on the highly stringent medium (-AHWL) when the three factors (eIF4G, eIF(iso)4G1, and eIF(iso)4G2) were coexpressed with BMYV-VPg (Fig. 3C). This result suggests that a possible weak interaction can occur between the 4G factors and BMYV-VPg. On the other hand, BMYV-P1- $C_{\text {term }}$ did not induce any self-activation of both reporter genes; however, no interaction between the $4 \mathrm{G}$ factors and this viral product was observed either (Fig. 3C).

Taken together, these data showed that the VPg-derived proteins of TuYV and BMYV exhibit a different pattern of interaction with the 4G and 4E factors: TuYV-VPg and TuYV-P1$\mathrm{C}_{\text {term }}$ only interacted in yeast with eIF(iso)4G1 from the $4 \mathrm{G}$ family whereas no interaction was recorded for any factor of the $4 \mathrm{E}$ family. Conversely, BMYV-VPg and BMYV-P1- $\mathrm{C}_{\text {term }}$ interacted with eIF4E1, eIF4E2, and eIF4E3 factors. No binding capacity of the BMYV-derived proteins with any of the $4 \mathrm{G}$ factors was detected.

\section{DISCUSSION}

The eukaryotic translation initiation factors eIF4F and eIF4G and their isoforms play a key role in plant susceptibility to RNA viruses (Le Gall et al. 2011; Robaglia and Caranta 2006; Truniger and Aranda 2009). In this study, we investigated the requirement of these factors for three phloem-restricted viruses (TuYV, BMYV, and BWYV-USA) belonging to the genus Polerovirus in the Luteoviridae family. We demonstrated that i) three closely related viruses belonging to the same viral genus (Polerovirus) rely on different translation initiation factors for their infection cycle and ii), similarly to the VPg encoding 
A
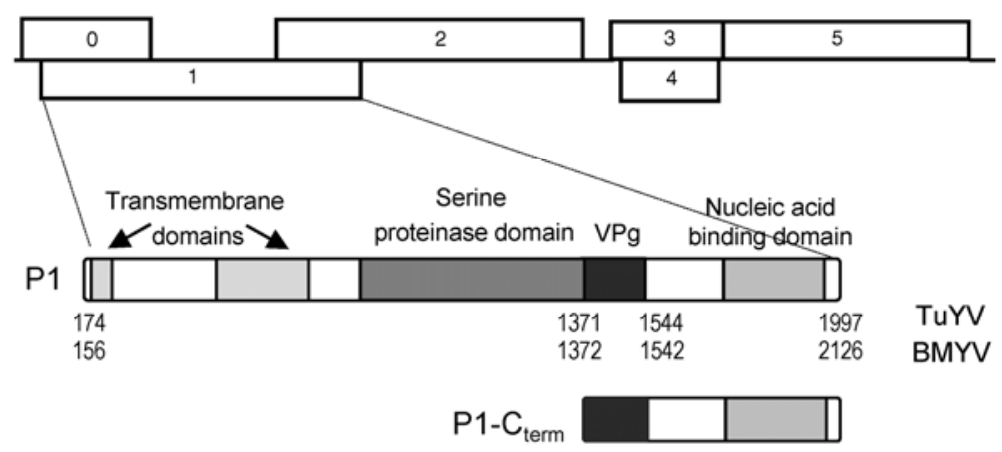

VPg
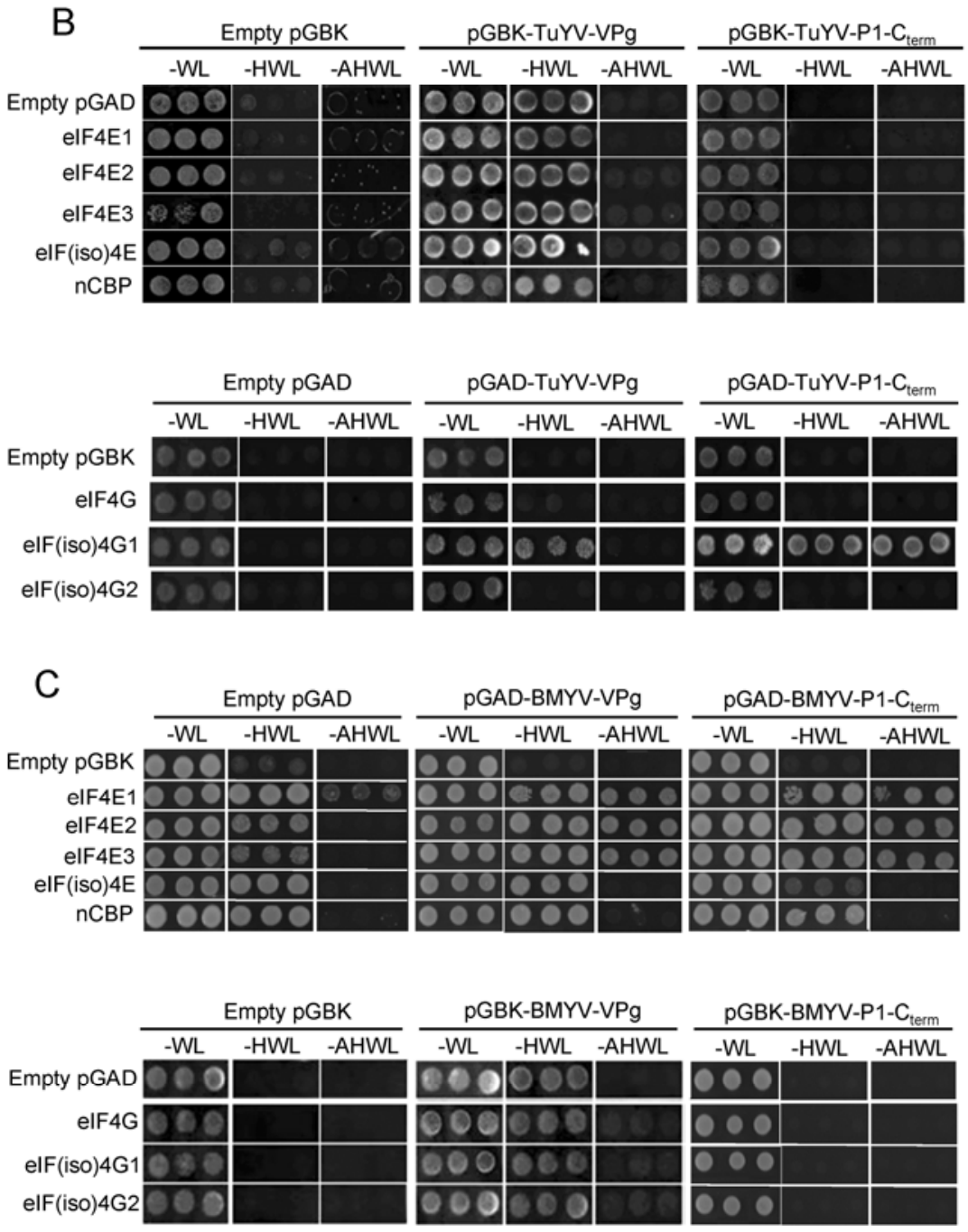

Fig. 3. Interaction assays between Turnip yellows virus (TuYV) or Beet mild yellowing virus (BMYV) viral genome-linked protein (VPg) and 4E or 4G isoforms using yeast two-hybrid system. A, Genome organization of poleroviruses. Rectangles represent the major open reading frames. The enlarged rectangle shows the P1 protein with the identified transmembrane, serine proteinase- and nucleic acid binding domains. Below are represented the C-terminal domain $\left(\mathrm{P} 1-\mathrm{C}_{\text {term }}\right.$ ) and the putative VPg (black rectangle). Viral genomic coordinates of the sequences cloned in the different yeast two-hybrid constructs are indicated for TuYV and BMYV. B, Upper panel: yeast interactions between TuYV-derived proteins and 4E factors. Yeast cells were co-transformed with pGBK:VPg or pGBK:P1-C term $_{\text {and }}$ one of the following constructs: pGAD:eIF4E1, pGAD:eIF4E2, pGAD:eIF4E3, pGAD:eIF(iso)4E, or pGAD:novel capbinding protein (nCBP) or with a set of negative controls. Cells doubly transformed were allowed to grow on a medium deprived of tryptophane and leucine $(-\mathrm{WL})$ before being transferred on selective media (deprived of histidine, tryptophane, and leucine [-HWL] or adenine, histidine, tryptophane, and leucine [-AHWL]). Yeast cells were allowed to grow for 3 to 5 days at $30^{\circ} \mathrm{C}$. Each combination is shown in triplicate. Lower panel: yeast interactions between TuYV-derived proteins and $4 \mathrm{G}$ factors. Yeast cells were co-transformed with pGAD:VPg or pGAD:P1-C term $_{\text {and }}$ one of the following constructs: pGBK: eIF4G, pGBK:eIF(iso)4G1, or pGBK:eIF(iso)4G2 or with a set of negative controls. The experiment was pursued as described above. C, Upper panel: yeast interactions between BMYV-derived proteins and $4 \mathrm{E}$ factors. Yeast cells were co-transformed with pGAD:VPg and one of the following constructs: pGBK:eIF4E1, pGBK:eIF4E2, pGBK:eIF4E3, pGBK:eIF(iso)4E, or pGBK:nCBP or with a set of negative controls. The experiment was pursued as described above. Lower panel: yeast interactions between BMYV-derived proteins and 4G factors. Yeast cells were co-transformed with pGBK:VPg or pGBK:P1$\mathrm{C}_{\text {term }}$ and one of the following constructs: pGAD:eIF4G, pGAD:eIF(iso)4G1, or pGAD:eIF(iso)4G2 or with a set of negative controls. The experiment was pursued as described above. The yeast expression and the size of all fusion proteins were checked by Western blot analysis (Supplementary Fig. S3). 
viruses requiring $4 \mathrm{E}$ or $4 \mathrm{G}$ factors, the VPg generated by poleroviruses is a potential viral determinant governing the specific interactions with these factors.

Several A. thaliana mutant lines impaired in the synthesis of eukaryotic translation initiation factors (eIF4G, eIF(iso)4G1, eIF(iso)4G2, eIF4E1, eIF4E2, eIF4E3, eIF(iso)4E, and nCBP) were inoculated with TuYV, BMYV, or BWYV-USA using viruliferous aphids. By using infectivity assays on A. thaliana mutants disrupted in each of the genes encoding eIF4G or eIF4E factors, we showed that TuYV, BMYV, and BWYVUSA exhibit different virus accumulation patterns in the collection of $A$. thaliana mutant lines. A strong reduction in TuYV accumulation was observed in the mutant disrupted for eIF(iso)4G1. The impact on TuYV structural proteins and RNA accumulation was important in At-eIF(iso)4Gl and AteIF(iso)4G1G2, whereas no effect on the virus titer was observed in the mutant affected only in eIF(iso)4G2 expression. This peculiar situation may suggest that the involvement of eIF(iso)4G2 in TuYV infectious process depends on the presence of the eIF(iso)4G1 isoform or that eIF(iso)4G2 is only active when eIF(iso)4G1 is absent. Additionally, the severe growth defect affecting the At-eIF(iso)4G1G2 line, in which both $4 \mathrm{G}$ isoforms are absent, may also account for the reduced TuYV titer due to pleotropic effects (Lellis et al. 2010).

The recruitment of eIF(iso)4G1 in the infectious process of TuYV is corroborated by yeast two-hybrid experiments which revealed a specific interaction between eIF(iso)4G1 and the predicted TuYV-VPg. TuYV-VPg, localized in the central domain of $\mathrm{P} 1$, is proteolytically released after cleavage by a serine proteinase-like domain protein ( $\mathrm{Li}$ et al. 2000, 2007; van der Wilk et al. 1997). Interestingly, a stronger interaction was observed between the $\mathrm{C}$-terminal domain of the $\mathrm{P} 1$ protein (P1- $\mathrm{C}_{\text {term }}$, encompassing the VPg-predicted sequence) and eIF(iso)4G1, suggesting that the VPg precursor may have a higher affinity for eIF(iso)4G1 than the VPg itself. Because the P1- $\mathrm{C}_{\text {term }}$ of PLRV displays nucleic acid binding properties, Prüfer and associates (1999) proposed that this domain may promote the formation of P1/viral RNA complexes required for the covalent binding of the VPg to the viral RNA. In the case of TuYV, binding of eIF(iso)4G1 to the $\mathrm{P} 1-\mathrm{C}_{\text {term }}$ precursor may participate in this step and initiate coupling between translation and viral replication.

A direct interaction between RYMV-VPg and the central domain of rice eIF(iso) $4 \mathrm{G} 1$ has been documented, and this interaction correlates with rice susceptibility (Hebrard et al. 2010). No further role of eIF4E factors was reported for this plant-virus system. It was also shown that potyviruses rely on a physical interaction between the VPg and factors of the eIF4E family for a successful plant infection (Le Gall et al. 2011). However, eIF4G factors were also shown to participate in host-potyvirus compatibility but their involvement was suspected to reflect a recruitment by the virus of the whole eIF(iso)4F complex (Nicaise et al. 2007). Finally, carmoviruses represent another peculiar case in which eIF4E factors, required for plant susceptibility, are suspected to interact with the viral RNA and not a viral protein (Diaz et al. 2004; Nieto et al. 2006). In this context, TuYV resembles RYMV because successful plant infection relies on a direct interaction between its VPg and eIF(iso)4G1.

The analysis of BMYV and BWYV-USA accumulation in the A. thaliana mutant lines pointed toward a different pattern of requirement of translation initiation factors. In contrast to TuYV, a significant reduction of both BMYV and BWYVUSA accumulation in eIF4E1-mutated plants was observed when compared with Col0, supporting a role of this translation initiation factor in their infection cycle. Among eIF4E factors, eIF4E1 played the most significant role in the virus infectious process, because no drastic variation of virus accumulation was observed in the A. thaliana lines impaired in the synthesis of eIF4E2 or eIF4E3. Involvement of eIF4E1 in BMYV and BWYV-USA multiplication cycles is also indirectly supported by a significantly higher virus accumulation in the At-eIF (iso) $4 E$ mutant line. Previous studies showed the ability of eIF4E protein to compensate for the lack of eIF(iso) $4 \mathrm{E}$ (Duprat et al. 2002). Therefore, a higher expression level of eIF4E1 in the At-eIF(iso) $4 E$ mutant could account for the virus titer elevation. Among the eIF4G mutant lines, only the double At-eIF(iso)4G1G2 mutant displayed a reproducible and significant reduction of both BMYV and BWYV-USA accumulation. This observation may reflect a functional redundancy between the $4 \mathrm{G}$ factors that can probably be recruited with the same efficiency by both viruses. But again, pleotropic effects due to the absence of both $4 \mathrm{G}$ isoforms in the At-eIF(iso) $4 G 1 G 2$ mutant (Lellis et al. 2010) may be responsible for the lower virus accumulation in this particular line.

Involvement of eIF4E1 factor in the BMYV infectious cycle was confirmed by the direct interaction between eIF4E1 and both the predicted BMYV-VPg and the C-terminal part of the P1 protein encompassing the VPg. Surprisingly, eIF4E2 and eIF4E3 were also able to interact with both viral proteins (BMYV-VPg and BMYV-P1-C $\mathrm{C}_{\text {term }}$ ) in the yeast two-hybrid system, although no requirement of these two factors was observed in plants. In addition to sharing a high sequence identity, eIF4E2 and eIF4E3 are poorly expressed in planta (Lellis et al. 2010). Therefore, a functional redundancy between both factors, or a lack of virus accessibility for these factors due to their low amount in planta, could explain why no difference in BMYV accumulation was observed in At-eIF4E2 and At-eIF4E3 mutants.

In summary, TuYV, BMYV, and BWYV-USA, three serologically and molecularly related viruses (de Miranda et al. 1995; Stevens et al. 2005), were shown to use different sets of translation initiation factors in the same host (A. thaliana). Infectivity analyses clearly showed that TuYV mainly relies on eIF(iso)4G1 whereas BMYV and BWYV-USA preferentially recruit eIF4E1. Moreover, this selective requirement of the translation initiation factors was supported by a direct interaction observed between the VPg-derived proteins and the identified translation initiation factors.

A selective recruitment of translation initiation factors in $A$. thaliana has previously been reported for potyviruses; however, different factors from the 4E family were required for virus infection: Tobacco etch virus, LMV, Turnip mosaic virus, and Plum pox virus rely on eIF(iso)4E for their infection cycle, whereas eIF4E1 is essential for Clover yellow vein virus multiplication in A. thaliana (Le Gall et al. 2011). The results reported in this work represent the first example of a selective recruitment of translation initiation factors from different families by viruses belonging to the same genus. By analogy with the resistance mechanism mediated by eIF4E for potyviruses or eIF(iso)4G1 for RYMV, polerovirus VPg, or a VPg-precursor protein might be the avirulence factor. No apparent sequence or structural similarities exist between potyvirus and polerovirus VPg except a high level of intrinsic disorder (Hebrard et al. 2009). This characteristic confers a high potential for interactions with multiple partners. Structural intrinsic disorder is also predicted for TuYV and BMYV-VPg (E. Hébrard, personal communication).

A complete resistance phenotype to TuYV, BMYV, or BWYV-USA was not observed in any of the mutant lines, suggesting a moderate level of functional redundancy between the different translation initiation factors for the infectious cycle of these viruses. Such a situation was already observed for some LMV isolates in lettuce (Candresse et al. 2002; German- 
Retana et al. 2000; Revers et al. 1997). It is noteworthy that BMYV host range is partially different and narrower than that of TuYV and BWYV-USA (Beuve et al. 2008; Graichen and Rabenstein 1996; Stevens et al. 1994). However, both BMYV and BWYV-USA are particularly well adapted to sugar beet, in contrast to TuYV (Beuve et al. 2008). Therefore, it is tempting to speculate that translation initiation factors might be involved in host range determination, as has been suggested for Melon necrotic spot virus, in which host susceptibility is governed by eIF4E (Nieto et al. 2011). However, additional experiments are required to confirm this hypothesis, such as generation of viral mutants in the VPg sequence and subsequent host range analysis.

\section{MATERIALS AND METHODS}

\section{Plant material.}

A. thaliana plants were grown in an environment-controlled chamber at $23^{\circ} \mathrm{C}$ (day) and $20^{\circ} \mathrm{C}$ (night) with a 10 -h light period. Two different $A$. thaliana lines were used to address eIF4E1 requirements in virus infectious cycle: At-eIF4E1 mutant (SALK145583) and cum1-1 (Yoshii et al. 2004), an ethylmethane sulphonate mutant provided by M. Ishikawa. Polerovirus infection assays were also performed on the following T-DNA insertion lines: At-eIF4E2 mutant (SALK103888), AteIF4E3 mutant (SALK101805), At- $n C B P$ mutant (SALK 053948), At-eIF4G mutant (SALK112882), At-eIF(iso)4G1 mutant (SALK009905), and At-eIF(iso)4G2 mutant (SALK 076633). Mutant At-eIF(iso) $4 E$ is a dSpm insertion mutant provided by C. Robaglia (Duprat et al. 2002). The double AteIF(iso)4G1G2 mutant was obtained after crossing of the single mutants SALK009905 and SALK076633, as described by Nicaise and associates (2007). The ecotype Col0 was used as a reference in the different experiments performed. Insertion of the T-DNA and absence of mRNA or protein expression were previously confirmed for all mutants used in this study (Duprat et al. 2002; Le Gall et al. 2011; Nicaise et al. 2007; Yoshii et al. 2004).

Virus inoculation by aphids and virus detection by ELISA.

A. thaliana plants were inoculated with TuYV, formerly referred to as BWYV-FL1 (Veidt et al. 1992), with BMYV-2ITB (Guilley et al. 1995) or with BWYV-USA (Beuve et al. 2008) using viruliferous Myzus persicae maintained on TuYV-, BMYV- or BWYV-USA-infected plants. Alternatively, virusfree aphids reared on Capsicum annuum were membrane-fed on purified virus, as described previously (Bruyère et al. 1997). Ten viruliferous aphids were transferred to each test plant for a 4-day inoculation period before being killed by an insecticide treatment. Because TuYV, BMYV, and BWYVUSA infections of $A$. thaliana plants remain symptomless, virus accumulation in systemic leaves was assessed 3 to 4 weeks after aphid inoculation by DAS-ELISA (Clark and Adams 1977) with a rabbit polyclonal antiserum raised against BMYV (Loewe, Kronach, Germany). In all, 5 to more than 60 plants per line were inoculated with TuYV, BMYV, or BWYV-USA using viruliferous aphids in a series of eight, six, and five independent experiments, respectively.

\section{Real-time RT-PCR.}

For qRT-PCR, total RNA was extracted (RNeasy plant mini kit; Qiagen, Courtaboeuf, France) 3 weeks postinoculation with TuYV from six Col0, six At-eIF4G, six At-eIF(iso)4G1, six At-eIF(iso)4G2, and 12 At-eIF(iso)4GlG2 plants. Primers were designed manually to amplify a 137-bp fragment from nucleotides 3,694 to 3,830 on the TuYV genome (accession number X13063). Total RNA (12.5 ng) in a $25-\mu$ l reaction were subjected to one-step RT-PCR using the Quantitect SYBR Green RT-PCR kit (Qiagen) containing two reverse transcriptases (Omniscript and Sensiscript) and a HotStartTaq DNA polymerase and the forward (BPqtF0; 5'-AAGACAATC TCGCGGGAAG-3') and reverse (BPqtR1; 5'-GGAGACGAA CTCCAAAATGAC- $3^{\prime}$ ) primers according to the manufacturer's recommendations. qRT-PCR was performed with the iCycler IQ system (Bio-Rad, Marnes-la-coquette, France). Reverse transcription was carried out at $50^{\circ} \mathrm{C}$ for $30 \mathrm{~min}$, followed by denaturation at $95^{\circ} \mathrm{C}$ for $15 \mathrm{~min}$. PCR cycles were as follows: 40 cycles each of $15 \mathrm{~s}$ at $94^{\circ} \mathrm{C}, 12 \mathrm{~s}$ at $55^{\circ} \mathrm{C}$, and $30 \mathrm{~s}$ at $72^{\circ} \mathrm{C}$. Real-time RT-PCR data were collected for $15 \mathrm{~s}$ at $78^{\circ} \mathrm{C}$ to avoid nonspecific fluorescence due to primer dimers occurring at low template concentrations. Absolute quantification was realized by using a dilution series from 103 to 108 viral RNA copies obtained from RNA extracted from purified TuYV virions. The $\mathrm{Ct}$ values were calculated by the Bio-Rad software and plotted proportionally to the logarithm of the input RNA copy numbers to generate the standard quantification curve. The correlation value was 0.999 and the amplification efficiency was 0.793 , calculated by the formula $E=\left[10^{-1 / \text { slope }}\right]-1$, with a slope of -3.942 . No fluorescent signal was obtained with noninfected A. thaliana plants and each analysis was carried out in duplicate.

\section{Construction of recombinant proteins for yeast two-hybrid assay.}

TuYV- and BMYV-VPg-encoded sequences were introduced in yeast shuttle vector pGADT7 and pGBKT7 (Clontech, Mountain View, CA, U.S.A.) as PCR fragments flanked by NdeI and BamHI or by NcoI and SalI restriction sites, respectively. The $5^{\prime}$ terminal nucleotide of TuYV-VPg was positioned at nucleotide 1,371 on the TuYV genome (accession number $\mathrm{X} 13063$ ) whereas the $3^{\prime}$ terminal nucleotide was located at nucleotide 1,535. However, due to cloning strategy, nine more nucleotides were added at the 3 ' end, positioning the last nucleotide of TuYV-VPg at nucleotide 1,544. BMYV-VPg sequence was positioned from nucleotide 1,372 to 1,542 of the BMYV sequence (accession number X83110). TuYV and BMYV-VPg contained one extra Methionine at their $5^{\prime}$ end. Additionally, the C-terminal domains of TuYV-P1 (nucleotide 1,371 to 1,997) or BMYV-P1 (nucleotide 1,372 to 2,126) proteins were introduced into both pGADT7 and pGBKT7 vectors for TuYV-P1$\mathrm{C}_{\text {term }}$ or only in pGBK vector for BMYV-P1-C $\mathrm{C}_{\text {term }}$.

Coding sequences of $A$. thaliana eIF4E1 (At4g18040), eIF4E2 (At1g29550), eIF4E3 (At1g29590), eIF(iso)4E (At5g35620), and nCBP (At5g18110), were cloned into pGADT7 yeast twohybrid vector in fusion with the GAL4 activation domain. Coding sequences of $A$. thaliana eIF4G (At3g60240), eIF(iso) 4G1 (At5g57870), and eIF(iso)4G2 (At2g24050) were cloned from $\mathrm{pET}$ vectors received from $\mathrm{K}$. Browning into the $\mathrm{pGBK}$ yeast two-hybrid vector in fusion with the GAL4 BD. Cloning of $4 \mathrm{G}$ or $4 \mathrm{E}$ factors into pGADT7 or pGBKT7, respectively, was performed starting with the corresponding pGBK- or pGAD-recombinant plasmids.

The AH109 (Ade- and His-) and Y187 yeast strains were transformed with the pGAD and the pGBK-derived constructs, respectively. The yeast double-hybrid assay used the Matchmaker GAL4 two-hybrid system 3 (Clontech) and was performed as previously described (Charron et al. 2008). Two to three independent experiments were performed.

\section{Expression of recombinant proteins in yeast.}

Yeast containing pGBK or pGAD:eIF4G isoforms together with pGBK: or pGAD:viral constructs were grown overnight in synthetic medium lacking tryptophan for pGBK-derived plasmids or leucine for pGAD-derived plasmids. Total proteins 
were prepared using the urea/sodium dodecyl sulfate (SDS) method, as described in the Clontech Yeast Protocol Handbook. Equalized loads of protein extracts were electrophoresed on an SDS-polyacrylamide gel and blotted onto Hybond ECL nitrocellulose membranes (GE Healthcare, Buckinghamshire, U.K.). Mouse c-Myc and hemagglutinin A monoclonal antibodies (Sigma-Aldrich. Saint-Quentin Fallavier, France) were used at a dilution 1/4,000 to detect pGBK- and pGAD-derived proteins, respectively. The secondary antibody was peroxidaselabeled rabbit antimouse serum (Sigma-Aldrich) used at a dilution 1/4,000. The chemiluminescent reaction was performed using either the Lumiglo reserve chemiluminescent substrate kit (KPL, Les Ulis, France) or the Lumi-Light ${ }^{\text {PLUS }}$-Western blotting substrate kit (Roche, Boulogne-Billancourt, France).

\section{ACKNOWLEDGMENTS}

We thank K. Browning (University of Texas, Austin, TX) for the eIF4G, eIF(iso)4G1, and eIF(iso)4G2 clones; C. Robaglia (Université de la Méditerranée, Marseille, France) for the At-eIF(iso)4E mutant; E. Klein and J.-L. Gallois for their technical help; and A. Miller (Iowa State University, Ames) for critically reading this manuscript. C. Reinbold and V. Brault were supported by a grant delivered by the INRA SPE (Santé des Plantes et Environnement) department. S. Lacombe and C. Caranta were supported by grants from the French national agency for research (ANR) in the frame of the MOVIe project (ANR-08-GENM-128). C. Reinbold, S. Lacombe, D. Scheidecker, L. Wiss, and M. Beuve contributed conception and realization of the experiments and data analysis; V. Ziegler-Graff and C. Caranta contributed data analysis, interpretation, and writing; and V. Brault contributed conception of the experiments, interpretation of data, statistical analysis, and writing.

\section{LITERATURE CITED}

Abdul-Razzak, A., Guiraud, T., Peypelut, M., Walter, J., Houvenaghel, M. C., Candresse, T., Le Gall, O., and German-Retana, S. 2009. Involvement of the cylindrical inclusion (CI) protein in the overcoming of an eIF4E-mediated resistance against Lettuce mosaic potyvirus. Mol. Plant Pathol.10:109-113.

Albar, L., Bangratz-Reyser, M., Hebrard, E., Ndjiondjop, M. N., Jones, M., and Ghesquiere, A. 2006. Mutations in the eIF(iso)4G translation initiation factor confer high resistance of rice to Rice yellow mottle virus. Plant J. 47:417-426.

Beauchemin, C., Boutet, N., and Laliberté, J. F. 2007. Visualization of the interaction between the precursors of VPg, the viral protein linked to the genome of Turnip mosaic virus, and the translation eukaryotic initiation factor iso $4 \mathrm{E}$ in planta. J. Virol. 81:775-782.

Beuve, M., Stevens, M., Liu, H. Y., Wintermantel, W. M., Hauser, S., and Lemaire, O. 2008. Biological and molecular characterization of an American sugar beet-infecting Beet western yellows virus isolate. Plant Dis. 92:51-60.

Bruyère, A., Brault, V., Ziegler-Graff, V., Simonis, M. T., Van den Heuvel, J. F., Richards, K., Guilley, H., Jonard, G., and Herrbach, E. 1997. Effects of mutations in the Beet western yellows virus readthrough protein on its expression and packaging and on virus accumulation, symptoms, and aphid transmission. Virology 230:323-334.

Candresse, T., Le Gall, O., Maisonneuve, B., German-Retana, S., and Redondo, E. 2002. The use of green fluorescent protein-tagged recombinant viruses to test Lettuce mosaic virus resistance in lettuce. Phytopathology 92:169-176.

Charron, C., Nicolai, M., Gallois, J. L., Robaglia, C., Moury, B., Palloix, A., and Caranta, C. 2008. Natural variation and functional analyses provide evidence for co-evolution between plant eIF4E and potyviral VPg. Plant J. 54:56-68.

Clark, M. F., and Adams, A. N. 1977. Characteristics of the microplate method of enzyme-linked immunosorbent assay for the detection of plant viruses. J. Gen. Virol. 34:475-483.

de Miranda, J. R., Stevens, M., de Bruyne, E., Smith, H. G., Bird, C., and Hull, R. 1995. Sequence comparison and classification of beet luteovirus isolates. Arch. Virol. 140:2183-2200.

Diaz, J. A., Nieto, C., Moriones, E., Truniger, V., and Aranda, M. A. 2004. Molecular characterization of a Melon necrotic spot virus strain that overcomes the resistance in melon and nonhost plants. Mol. PlantMicrobe Interact. 17:668-675.

Duprat, A., Caranta, C., Revers, F., Menand, B., Browning, K. S., and
Robaglia, C. 2002. The Arabidopsis eukaryotic initiation factor (iso)4E is dispensable for plant growth but required for susceptibility to potyviruses. Plant J. 32:927-934.

Gallie, D. R., and Browning, K. S. 2001. eIF4G functionally differs from eIFiso4G in promoting internal initiation, cap-independent translation, and translation of structured mRNAs. J. Biol. Chem. 276:36951-36960.

Gallois, J. L., Charron, C., Sanchez, F., Pagny, G., Houvenaghel, M. C., Moretti, A., Ponz, F., Revers, F., Caranta, C., and German-Retana, S. 2010. Single amino acid changes in the Turnip mosaic virus viral genome-linked protein (VPg) confer virulence towards Arabidopsis thaliana mutants knocked out for eukaryotic initiation factors eIF(iso)4E and eIF(iso)4G. J. Gen. Virol. 91:288-293.

German-Retana, S., Candresse, T., Alias, E., Delbos, R. P., and Le Gall, O. 2000. Effects of green fluorescent protein or beta-glucuronidase tagging on the accumulation and pathogenicity of a resistance-breaking Lettuce mosaic virus isolate in susceptible and resistant lettuce cultivars. Mol. Plant-Microbe Interact. 13:316-324.

Goodfellow, I. G., and Roberts, L. O. 2008. Eukaryotic initiation factor 4E. Int. J. Biochem. Cell Biol. 40:2675-2680.

Graichen, K., and Rabenstein, F. 1996. European isolates of Beet western yellows virus (BWYV) from oilseed rape (Brassica napus L. spp. napus) are non-pathogenic on sugar beet (Beta vulgaris L. var. altissima) but represent isolates of Turnip yellows virus (TuYV). J. Plant Dis. Prot. 103:233-245.

Guilley, H., Richards, K. E., and Jonard, G. 1995. Nucleotide sequence of Beet mild yellowing virus RNA. Arch. Virol. 140:1109-1118.

Hebrard, E., Pinel-Galzi, A., and Fargette, D. 2008. Virulence domain of the RYMV genome-linked viral protein VPg towards rice rymv1-2mediated resistance. Arch. Virol. 153:1161-1164.

Hebrard, E., Bessin, Y., Michon, T., Longhi, S., Uversky, V. N., Delalande, F., Van Dorsselaer, A., Romero, P., Walter, J., Declerck, N., and Fargette, D. 2009. Intrinsic disorder in viral proteins genome-linked: Experimental and predictive analyses. Virol. J. 6:23.

Hebrard, E., Poulicard, N., Gerard, C., Traore, O., Wu, H. C., Albar, L., Fargette, D., Bessin, Y., and Vignols, F. 2010. Direct interaction between the Rice yellow mottle virus (RYMV) VPg and the central domain of the rice eIF(iso)4G1 factor correlates with rice susceptibility and RYMV virulence. Mol. Plant-Microbe Interact. 23:1506-1513.

Hjulsager, C. K., Olsen, B. S., Jensen, D. M., Cordea, M. I., Krath, B. N., Johansen, I. E., and Lund, O. S. 2006. Multiple determinants in the coding region of Pea seed-borne mosaic virus $\mathrm{P} 3$ are involved in virulence against sbm-2 resistance. Virology 355:52-61.

Jiang, J., and Laliberte, J. F. 2011. The genome-linked protein VPg of plant viruses : A protein with many partners. Curr. Opin. Virol. 1:347354.

Kanyuka, K., Druka, A., Caldwell, D. G., Tymon, A., McCallum, N., Waugh, R., and Adams, M. J. 2005. Evidence that the recessive bymovirus resistance locus rym4 in barley corresponds to the eukaryotic translation initiation factor 4E gene. Mol. Plant Pathol. 6:449-458.

Khan, M. A., Miyoshi, H., Ray, S., Natsuaki, T., Suehiro, N., and Goss, D. J. 2006. Interaction of genome-linked protein (VPg) of Turnip mosaic virus with wheat germ translation initiation factors eIFiso4E and eIFiso4F. J. Biol. Chem. 281:28002-28010.

Lee, J. H., Muhsin, M., Atienza, G.A., Kwak, D. Y., Kim, S. M., De Leon, T. B., Angeles, E. R., Coloquio, E., Kondoh, H., Satoh, K., Cabunagan, R. C., Cabauatan, P.Q., Kikuchi, S., Leung, H., and Choi, I. R. 2010. Single nucleotide polymorphisms in a gene for translation initiation factor (eIF4G) of rice (Oryza sativa) associated with resistance to Rice tungro spherical virus. Mol. Plant-Microbe Interact. 23:29-38.

Le Gall, O., Aranda, M. A., and Caranta, C. 2011. Plant resistance to viruses mediated by translation initiation factors. Pages 177-194 in: Recent Advances in Plant Virology. C. Caranta, M. A. Aranda, M. Tepfer, and J. J. Lopez-Moya, eds. Caister Academic Press, Norwich, U.K.

Lellis, A. D., Allen, M. L., Aertker, A. W., Tran, J. K., Hillis, D. M., Harbin, C. R., Caldwell, C., Gallie, D. R., and Browning, K. S. 2010. Deletion of the eIFiso4G subunit of the Arabidopsis eIFiso4F translation initiation complex impairs health and viability. Plant Mol. Biol. 74:249-263.

Li, X., Ryan, M. D., and Lamb, J. W. 2000. Potato leafroll virus protein P1 contains a serine proteinase domain. J. Gen. Virol. 81:1857-1864.

Li, X., Halpin, C., and Ryan, M. D. 2007. A novel cleavage site within the Potato leafroll virus P1 polyprotein. J. Gen. Virol. 88:1620-1623.

Marcotrigiano, J., Gingras, A. C., Sonenberg, N., and Burley, S. K. 1999. Cap-dependent translation initiation in eukaryotes is regulated by a molecular mimic of eIF4G. Mol. Cell 3:707-716.

Mayo, M. A., and Ziegler-Graff, V. 1996. Molecular biology of luteoviruses. Adv. Virus Res. 46:413-460.

Mayo, M. A., Barker, H., Robinson, D. J., Tamada, T., and Harrison, B. D. 1982. Evidence that Potato leafroll virus is positive stranded, is linked to a small protein and does not contain polyadenylate. J. Gen. Virol. 59:163-167. 
Miyoshi, H., Suehiro, N., Tomoo, K., Muto, S., Takahashi, T., Tsukamoto, T., Ohmori, T., and Natsuaki, T. 2006. Binding analyses for the interaction between plant virus genome-linked protein (VPg) and plant translational initiation factors. Biochimie 88:329-340.

Mutterer, J. D., Stussi-Garaud, C., Michler, P., Richards, K. E., Jonard, G., and Ziegler-Graff, V. 1999. Role of the Beet western yellows virus readthrough protein in virus movement in Nicotiana clevelandii. J. Gen Virol. 80:2771-2778.

Nicaise, V., Gallois, J. L., Chafiai, F., Allen, L. M., Schurdi-Levraud, V., Browning, K. S., Candresse, T., Caranta, C., Le Gall, O., and GermanRetana, S. 2007. Coordinated and selective recruitment of eIF4E and eIF4G factors for potyvirus infection in Arabidopsis thaliana. FEBS (Fed. Eur. Biochem. Soc.) Lett. 581:1041-1046.

Nieto, C., Morales, M., Orjeda, G., Clepet, C., Monfort, A., Sturbois, B., Puigdomenech, P., Pitrat, M., Caboche, M., Dogimont, C., Garcia-Mas, J., Aranda, M. A., and Bendahmane, A. 2006. An eIF4E allele confers resistance to an uncapped and non-polyadenylated RNA virus in melon. Plant J. 48:452-462.

Nieto, C., Rodriguez-Moreno, L., Rodriguez-Hernandez, A. M., Aranda, M. A., and Truniger, V. 2011. Nicotiana benthamiana resistance to nonadapted Melon necrotic spot virus results from an incompatible interaction between virus RNA and translation initiation factor 4E. Plant J. 66:492-501.

Prüfer, D., Kawchuk, L., Monecke, M., Nowok, S., Fischer, R., and Rohde, W. 1999. Immunological analysis of Potato leafroll luteovirus (PLRV) P1 expression identifies a $25 \mathrm{kDa}$ RNA-binding protein derived via P1 processing. Nucleic Acids Res. 27:421-425.

Revers, F., Lot, H., Souche, S., Le Gall, O., Candresse, T., and Dunez, J. 1997. Biological and molecular variability of Lettuce mosaic virus isolates. Phytopathology 87:397-403.

Robaglia, C., and Caranta, C. 2006. Translation initiation factors: A weak link in plant RNA virus infection. Trends Plant Sci. 11:40-45.
Stein, N., Perovic, D., Kumlehn, J., Pellio, B., Stracke, S., Streng, S., Ordon, F., and Graner, A. 2005. The eukaryotic translation initiation factor $4 \mathrm{E}$ confers multiallelic recessive Bymovirus resistance in Hordeum vulgare (L.). Plant J. 42:912-922.

Stevens, M., Smith, H. G., and Hallsworth, P. B. 1994. The host range of beet yellowing viruses among common arable weed species. Plant Pathol. 43:579-588.

Stevens, M., Freeman, B., Liu, H. Y., Herrbach, E., and Lemaire, O. 2005. Beet poleroviruses: Close friends or distant relatives? Mol. Plant Pathol.6:1-9.

Treder, K., Kneller, E. L., Allen, E. M., Wang, Z., Browning, K. S., and Miller, W. A. 2008. The 3' cap-independent translation element of Barley yellow dwarf virus binds eIF4F via the eIF4G subunit to initiate translation. RNA 14:134-147.

Truniger, V., and Aranda, M. A. 2009. Recessive resistance to plant viruses. Adv. Virus Res. 75:119-159.

van der Wilk, F., Verbeek, M., Dullemans, A. M., and van den Heuvel, J. F. 1997. The genome-linked protein of Potato leafroll virus is located downstream of the putative protease domain of the ORF1 product. Virology 234:300-303.

Veidt, I., Bouzoubaa, S. E., Leiser, R. M., Ziegler-Graff, V., Guilley, H. Richards, K., and Jonard, G. 1992. Synthesis of full-length transcripts of Beet western yellows virus RNA: Messenger properties and biological activity in protoplasts. Virology 186:192-200.

Wittmann, S., Chatel, H., Fortin, M. G., and Laliberte, J.F. 1997. Interaction of the viral protein genome linked of Turnip mosaic potyvirus with the translational eukaryotic initiation factor (iso) $4 \mathrm{E}$ of Arabidopsis thaliana using the yeast two-hybrid system. Virology 234:84-92.

Yoshii, M., Nishikiori, M., Tomita, K., Yoshioka, N., Kozuka, R., Naito, S., and Ishikawa, M. 2004. The Arabidopsis cucumovirus multiplication 1 and 2 loci encode translation initiation factors $4 \mathrm{E}$ and $4 \mathrm{G}$. J. Virol. 78:6102-6111. 